\title{
A wind-turbine for autonomous stations for radio detection of neutrinos
}

\author{
Anna Nelles* for the ARIANNA Collaboration ${ }^{\dagger}$ \\ ECAP, Friedrich-Alexander-Universität Erlangen-Nürnberg, 91058 Erlangen, Germany \\ DESY, Platanenallee 6, 15738, Zeuthen, Germany \\ E-mail: anna.nelles@desy.de
}

\begin{abstract}
A future large radio array for the detection of neutrinos may need to be built in remote areas without access to a power grid, such as the Antarctic ice-shelf or Greenland. In such a scenario lifetime will need to be optimized by combining different renewable and autonomous power sources. The ARIANNA experiment on the Ross-Ice-Shelf has run stably on solar power during the Austral summer, as soon as the Sun was more than 3 degrees above the horizon. However, the dark months of polar winter provide essential lifetime, especially with respect to multi-messenger studies. We will present a wind-turbine that has been custom-designed for the ARIANNA experiment to be radio-quiet, sensitive to low winds and able to sustain extreme Antarctic weather. The first important milestone was achieved in 2018 when a first wind turbine survived the winter months and powered a station for $24 \%$ of the winter time. We will report on the in-situ performance of the second-generation turbine already reaching $39 \%$ uptime during the weak summer winds and the perspective of this technique.
\end{abstract}

36th International Cosmic Ray Conference -ICRC2019-

July 24th - August 1st, 2019

Madison, WI, U.S.A.

\footnotetext{
* Speaker.

${ }^{\dagger}$ for collaboration list see PoS(ICRC2019)1177
} 


\section{Introduction}

Radio detection of neutrinos delivers the promise of being able to sparsely instrument large volumes of ice and turn them into a detector for neutrinos above PeV energies. Instrumenting large volumes is needed to detect potentially very low fluxes [1]. While scientifically promising, it also comes at the cost of a logistics burden. Remote polar areas, with few exceptions such as South Pole, do not host an infrastructure powerful enough to power a grid of detectors at distances of many kilometers from the station. This, along with sustainability of green energy, argues for remote power generation. While solar panels have shown to operate well, they are unable to power stations through the long polar winters. Batteries are often unsuitable to withstand cold temperatures or become too large when needed to bridge months of complete darkness.

Using power generated in wind turbines seems the logical approach. However, experience has not always been positive. Not many off-the-shelf wind-turbines have been designed for harsh polar climates. Bearings and greases freeze and the turbines are often destroyed during storms. Turbines designed for cold temperatures generally need high winds to turn on, not delivering the lifetime expected. Also, anchoring wind turbines to the ground proves difficult for large turbines given icy underground and non-existent concrete facilities.

Radio detection of neutrino also comes with an additional peculiarity. Steering electronics or connecting brushes often create radio-frequency (RF) noise that disturbs the search for cosmic signals, which are expected to be just above the thermal radio background. Thus, all electronics have to be developed and tested to be RF quiet, especially AC-DC or DC-DC converters or transformers should be avoided where ever possible or grounded and heavily shielded. Not having a defined electrical ground, due to the icy underground provides additional challenges. In combination with the very dry air, the build-up of static charges and their discharges can already cause measurable signals.

Despite the challenges, the ARIANNA site at Moore's Bay [2] has successfully been used for testing and development of a wind-turbine system. These proceedings report on the progress, experiences and future developments and opportunities.

\section{Design of the wind-turbine}

Performance and requirements for a wind-turbine depend heavily on the location of the experiment. The latitude of the experiment determines the time in which complete darkness is present and power has to come solely from wind-turbines. Further considerations are the elevation above sea-level that determines the average air pressure, local temperatures and wind-speeds that both impose technical restrictions on size and material.

In general, for RF quietness and robustness one would like to avoid as many steering elements as possible naturally leading to the choice of a vertical-axis wind turbine. While not as powerful as the ones having their rotational axis aligned with the horizon and vertical blades, they are much more robust to storms as the changing directions do not create horizontal torque on the bearings and no electronics are required to brake and steer the rotor. 


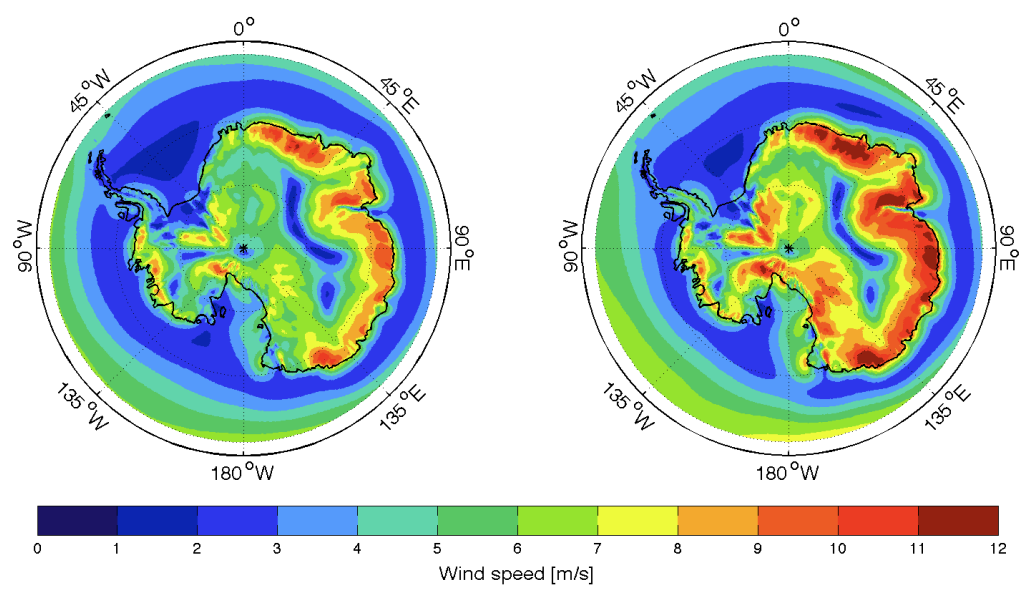

Figure 1: Average windspeed for Antarctica during summer on the left and during winter shown on the right. This figure illustrates that quite large variations are observed as function of location on the continent. From [8].

\subsection{Wind conditions at sites}

For the case of radio detection of neutrinos, we consider three potential sites. South Pole, the location of IceCube [3], a future IceCube-Gen2 [4], and ARA [5], Moore's Bay on the Antarctica ice-shelf, the site of ARIANNA [6], as well as Summit station in Greenland, as potential site in the North [7]. As illustrated as an example for Antarctica in Fig. 1 wind speeds vary across locations and as function of height above ground. For the performance of a wind turbine, one also needs to consider periods without any wind and those where the wind is so strong that not all power can be used. Optimal are sites with continuous wind of medium strength.

Fig. 2 shows the average wind power density at the three considered sites as function of time in a year. Data from historical records has been used. These, of course, only provide an indication, as with respect to changing conditions caused by climate change. As additional data was available, we also show the difference for two heights above sea-level for the same site. While not fully accurate for all sites, this illustrates how one could compensate at a site with poorer wind conditions by installing higher towers. Notwithstanding that a high tower increases the logistics burden.

Fig. 3 shows the fraction of time in which a certain wind speed is measured. It can be seen for example that Summit has periods with very high winds, but also a larger variability with periods of no wind. When interpreting this figure, one should note that a single day without wind is not as problematic for the lifetime, as several continuous days without wind, as the first is easily bridged by a battery. Estimating a lifetime fraction depends also on the battery size and therefore requires a full simulation or in-situ testing and an estimate based on average numbers can only give an indication of the performance.

\subsection{The Savant series}

For the ARIANNA site a custom-designed wind turbine was built and continuously developed over the past three years (see Fig. 4). The turbine has a newly developed geometry based on the 




Figure 2: Average wind power density per month for the sites at Moore's Bay (two heights above sea level), South Pole and Summit Station. One should note that Summit Station is on the Northern hemisphere, which is why a phased shift is observed.



Figure 3: Histogram of observed wind speeds at three different sites during local winter, where power from a wind turbine may be relevant.

traditional Savonius turbine. It is called twisted Savonius as two displaced half circle arc crosssections, in the horizontal plane, are swept vertically with an increasing azimuth around the axis of rotation. The new geometry is technically accomplished by first cutting a cylinder with a twisted cut then intertwining the two twisted cuts in such a manner that a partial twist, in the range of $90^{\circ}$, is accomplished. This twist helps to reduce vibrations, noise and fluctuations in torque. Above all, it renders the turbine aerodynamically self starting, which is not the case for a traditional straight Savonius.

Integrated within the turbine is the permanent magnet rotor of an air gap winding synchronous 
generator. This allows for full electrical control, through the loading of the generator by the rotational speed of the turbine. Furthermore, the generator is designed for a low load angle which enables the robust and high efficiency rectification with diodes. Thus, when used to charge a battery, the rotational speed of the turbine, and thus the delivered voltage, can be controlled with one single passively controlled over-voltage-protection circuit. This reduces system complexity, potential electronic interference (RFI) and increases the robustness of the turbine, a high-priority in polar climate.

Up to date, three versions of the Savant turbine have been installed at the ARIANNA site at Moore's Bay, Antarctica. In December 2016, Savant 1 was installed as Twisted Savonius with dimensions of $0.4 \times 0.2 \mathrm{~m}^{2}$. The system was equipped with DC protection and a commercial direct drive permanent magnet synchronous generator (PMSG), which ran as low-efficiency generator and transformer. In 2017 (Savant 2), the system was upgraded to use a air gap winding PMSG designed specifically for the Savant turbine as "0-0-loss" since it had no transformer. AC-protection circuits were added and the top mount was slimmed to improve aerodynamic properties. Finally in 2018, a bigger turbine was installed with dimensions $0.6 \times 0.3 \mathrm{~m}^{2}$ and a weight of $10.5 \mathrm{~kg}$. We included both a DC and AC protection system and a resistive grounding system to ensure minimal RFI contamination.
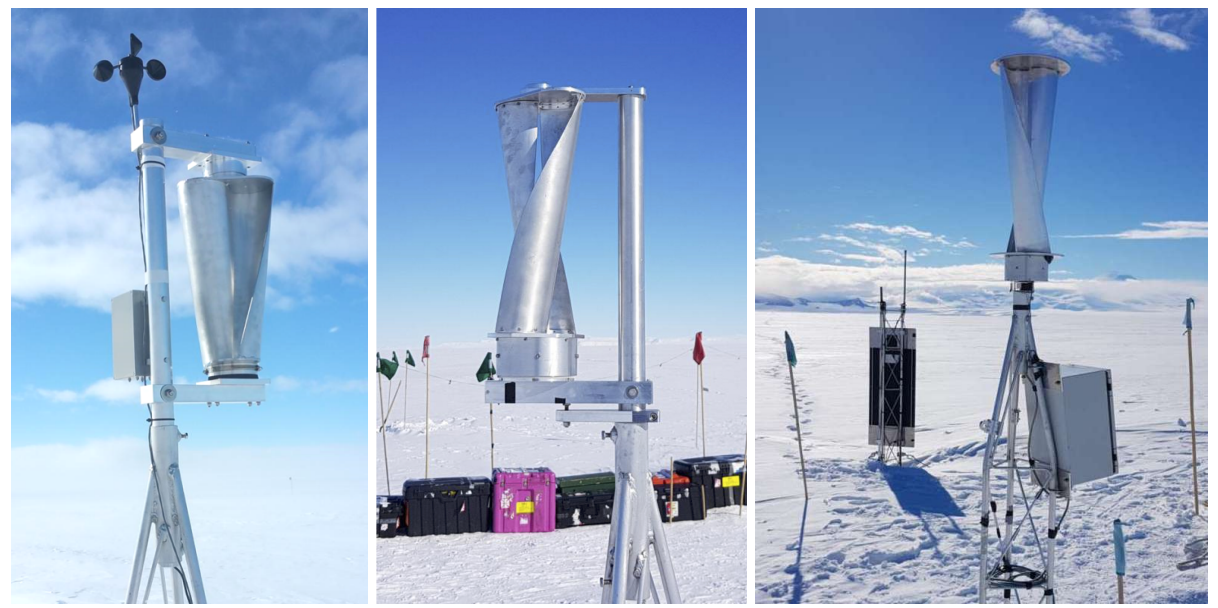

Figure 4: Wind turbines as installed at the ARIANNA site. Left: the first generation turbine, installed December 2016. It was never connected to a station and only used to test whether it survived the winter and delivered power. Middle: the second generation turbine installed in December 2017. It was connected to a station and had improved electronics. Right: The third generation turbine, larger in size, installed in December 2018. It is the sole power source of the station, allowing to measure year-round performance without a solar panel as main power source.

\section{Performance in the field}

All wind turbines as shown in Fig. 4 delivered power in the polar conditions at Moore's Bay. Savant 1 and 2 were retrieved fully working after a season in the field. That said, contact to the station powered by the wind turbine Savant 3 has been lost in March after four months of operation. It is still unclear whether this is related to a turbine or communications failure. The 


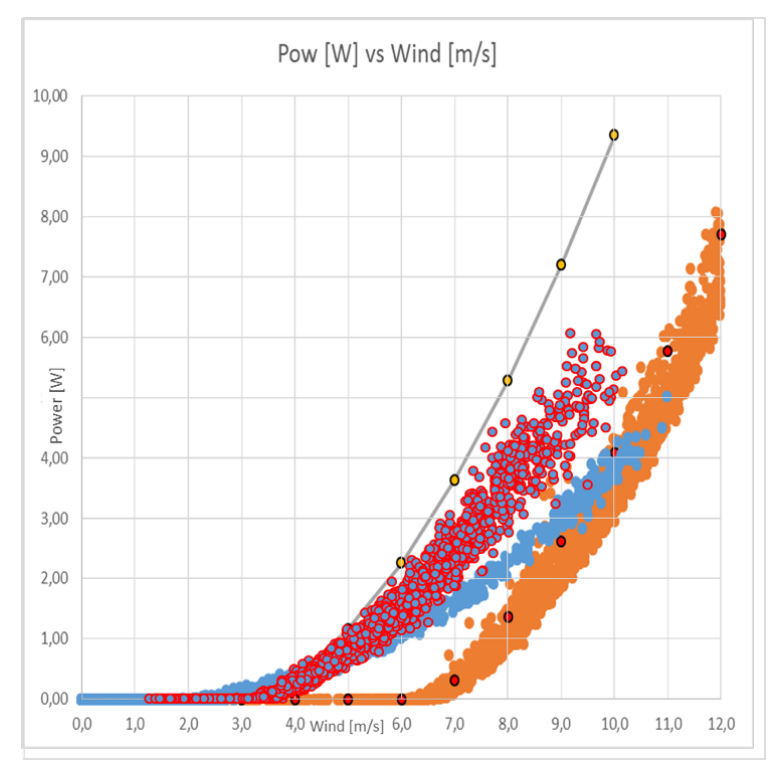

Figure 5: Power delivered by wind turbine. Overlaid are simulations for two versions of the turbine: Red dots with black outline (original version, Savant 1), as well as yellow dots with black outline (improved version, Savant 2). Data taken in the field from the first installation is shown in orange, matching the simulation (red+black). Data from the second year is shown both in raw format (blue) and load corrected (blue with red outline).

working hypothesis is damage to the battery charging unit caused by the usage of a Zener diode to reduce RF noise, however other failure modes remain plausible. Due to the long turn-around times in Antarctica, the site can only be visited again in the coming December to clarify the issue at hand. The performance of the turbine is monitored independently of the station, so we will be able to retrieve data next summer and assess the situation.

Fig. 5 shows the recorded power values compared to simulated power values of Savant 1 and Savant 2. While the values for Savant 1 agree well between measurement and simulations, the performance of Savant 2 is a bit worse than anticipated, which has been attributed to the electrical components after the turbine. These have been upgraded in Savant 3 (along with an increase in size), but data has yet to be collected from Moore's Bay for a detailed comparison.

Fig. 6 shows the delivered voltage at station level as function of time for Savant 2 during the Antarctic winter months, where the Sun never rises above the horizon. When reaching values of 13.5 the battery is charged, meaning that the wind turbine provided sufficient power for the entire system and fully charged the battery. An uptime of $24 \%$ was achieved wit Savant 2. Before loosing contact, Savant 3 powered the station in the weak summer winds, achieving 39\% uptime already (see Fig. 7).

\section{Future developments}

The detailed developments will depend on the site chosen for a neutrino array. We have calculated achievable uptime fractions for different sizes of turbines and battery capacities. With a turbine of the size of Savant 3, uptimes of up to $85 \%$ are possible in good wind conditions and 


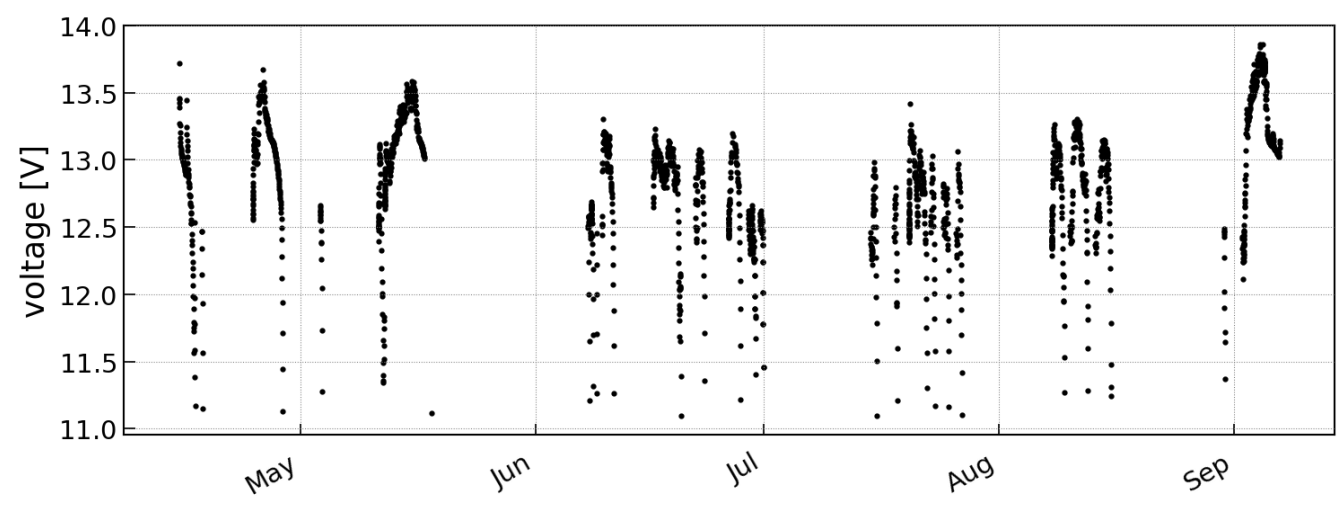

Figure 6: Voltage recorded at the ARIANNA station equipped with the wind turbine Savant 2 during the polar winter of 2018. The station showed an uptime of $24 \%$ during the winter, showing potential for achieving a considerable amount of lifetime during the winter with wind power.

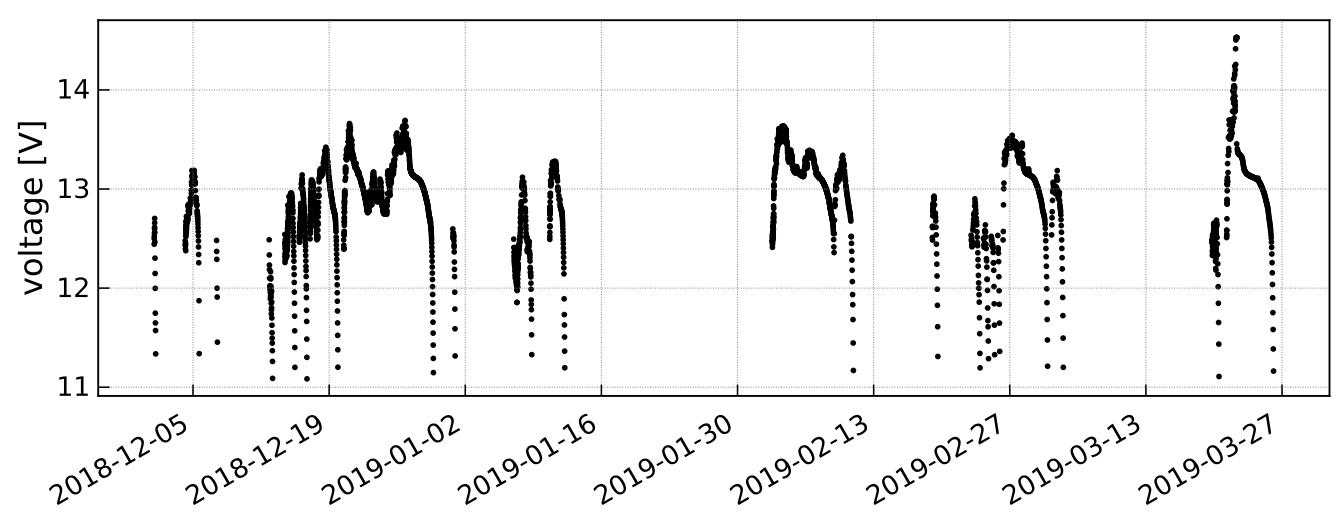

Figure 7: Voltage recorded at the ARIANNA station equipped with the wind turbine Savant 3 between December 2018 and March 2019. The station showed an uptime of 39\% during the summer months with in general lower wind speeds.

with a battery of $1800 \mathrm{Wh}$ capacity. Increasing the capacity does not significantly add livetime at some point. Instead the size of the turbine needs to be increased to generate power in low wind situations. With a turbine of $1 \mathrm{~m}^{2}$ area uptimes of $99 \%$ seem achievable.

In case of a large array with spacing of the order of kilometers between stations, cabling the array for power may not be an option. Even in case that cabling will be necessary, if for example wifi data transmission were to interfere with other experiments, power from a station may not be available in sufficient quantities. For both these scenarios, a combination of windturbine, solar panels and batteries may power the station more than $90 \%$ of the time during winter providing $100 \%$ uptime during the summer. However, sites with weak wind conditions may require significantly larger turbines, which increases the logistics burden, which adds another parameter to the site decision matrix. 


\section{Conclusion}

We have presented our work regarding the power generation for autonomous stations in a radio neutrino array. The ARIANNA Collaboration has successfully deployed and tested a customdesigned wind turbine at Moore's Bay in Antarctica. Uptimes of 39\% have been achieved with the current combination of battery capacity and wind turbine size. We estimate that it is feasible to achieve at least $95 \%$ livetime for a radio array running solely on renewable energies by scaling the turbine and battery size.

\section{Acknowledgements}

We are grateful to the U.S. National Science Foundation-Office of Polar Programs, the U.S. National Science Foundation-Physics Division (grant NSF-1607719) and the U.S. Department of Energy. We thank generous support from the German Research Foundation (DFG), grant NE 2031/2-1 and GL 914/1-1, the Taiwan Ministry of Science and Technology. We acknowledge support from the Uppsala university Vice-Chancellor's travel grant (sponsored by the Knut and Alice Wallenberg Foundation) and the C.F. Liljewalch travel scholarships. We are thankful for support from the MEPhI Academic Excellence Project (Contract No. 02.a03.21.0005) and the Megagrant 2013 program of Russia, via agreement 14.12.31.0006 from 24.06.2013.

With regard to wind-turbine development we are thankful for support from the Swedish Government strategic program 'Stand Up for Energy' and from the Helmholtz-W2 professorship recruitment funds.

\section{References}

[1] A. Vieregg et al., Astrophysics Uniquely Enabled by Observations of High-Energy Cosmic Neutrinos, Astro2020, in BAAS, vol. 51, p. 185, May 2019

[2] A. Anker et al., Targeting ultra-high energy neutrinos with the ARIANNA experiment, Advances in Space Research, in press.

[3] IceCube Collaboration: M. G. Aartsen et al., Astrophysical Neutrinos and Cosmic Rays Observed by IceCube, Advances in Space Research 62 (2018) 2902-2930

[4] M. G. Aartsen et al., Neutrino astronomy with the next generation IceCube Neutrino Observatory, Astro 2020 Decadal survey

[5] P. Allison et al., Design and Initial Performance of the Askaryan Radio Array Prototype EeV Neutrino Detector at the South Pole, Astropart. Phys., vol. 35, p. 457, 2012.

[6] S.W. Barwick et al.,A First Search for Cosmogenic Neutrinos with the ARIANNA Hexagonal Radio Array, Astropart. Phys. 70 (2015), 12-26

[7] C. Deaconu et al., Measurements and Modeling of Near-Surface Radio Propagation in Glacial Ice and Implications for Neutrino Experiments, Phys.Rev. D98 (2018) no.4, 043010

[8] C. Hallgren, Assessment of green power production in Antarctica, 2013, urn:nbn:se:uu:diva-341702 